\title{
Dual-Band, Dual-Polarized Two Element Slot Antenna for Fifth Generation Mobile Devices
}

\author{
Jitendra Vaswani ${ }^{a}$, Archana Agarwal ${ }^{\text {b }}$ \\ a,b Electronics and Communication Engineering Department Sangam University, Bhilwara, India \\ aitendra2104@ gmail.com, ${ }^{\mathrm{b}}$ archana.agrawal@ sangamuniversity.ac
}

Article History: Received: 10 November 2020; Revised 12 January 2021 Accepted: 27 January 2021; Published online: 5 April 2021

\begin{abstract}
This paper presents a two-element dual-band MIMO antenna with dual-polarization for 5G user equipment. Both operating frequencies are of sub- $6 \mathrm{GHz}$ band centered at $3.6 \mathrm{GHz}$ and $5.5 \mathrm{GHz}$ respectively and are independent of each other. Antenna polarizations depend on the placement of the feed elements on the PCB. The antenna is designed on FR-4 substrate to ensure its easy availability and keeping it cost-effective. The resultant radiation pattern of the antenna set is bi-directional with good gain and efficiency.
\end{abstract}

Keywords: 5G, WLAN, MIMO, Sub-6 GHz, Dual-band, Dual-polarization

\section{Introduction}

In the first decade of the 20th century, the internet over mobile phones was an additional feature as the world was in a transition phase of going from offline to online and mobile phones were primarily used for voice calls. Internet was primarily accessed over computers and laptops using LAN, Wi-Fi or plug-in devices. But, in the second decade, most of the services went online, generation of mobile telephony changed from $2 \mathrm{G}$ to $3 \mathrm{G}$ to $4 \mathrm{G}$, increasing the data speed from a few kbps to several Mbps. Mobile phones are now not only used for voice calls but for HD video calls, streaming live events, and much more. IoT also emerged during this time, interconnecting devices via the internet and providing remote access to them. These innovations needed high internet speed without losing mobility. Currently, we are using $4 \mathrm{G}$ services and research is going on for developing $5 \mathrm{G}$ standards to provide even high data speeds.

Multi-band, multi-standard, high efficiency, and low specific absorption rate (SAR) antenna are essential for antenna assembled in mobile phones. The author has also mentioned the dimensions of few smartphones which one should consider while designing antenna for smartphones.[1] A multi-band (2G, 3G, 4G, and 5G) dualpolarized antenna for an indoor mobile base station is proposed by the author.[2] An eight-port MIMO antenna system is designed for $5 \mathrm{G}$ applications operating in the frequency range of 3.3 to $4.2 \mathrm{GHz}$, with good isolation (better than-14.4 dB) and gain varying with the frequency between 3 to $5 \mathrm{dBi}$.[3] An eight loop antenna system is proposed for a $5 \mathrm{G}$ smartphone of the screen size of 5 inches, with FR-4 substrate and 8 antennas are placed in two groups of five antennas and three antennas. The value ECC (Envelope Correlation Coefficient) of any two antennas was less than 0.2 with an efficiency of around $40 \%$ and the over gain for the antenna is $2 \mathrm{dBi}$. [4] A metal-frame-integrated MIMO system with eight-antennas that operates in LTE (long term evolution) bands $(2.496 \mathrm{GHz}-2.69 \mathrm{GHz})$ and $5 \mathrm{G}$ band $(3.4 \mathrm{GHz}-3.8 \mathrm{GHz})$ for future smartphones is proposed. The antenna design was simulated, experimentally tested and good efficiency $(>44 \%)$ and low ECC $(<0.2)$ was discovered. [5] An array of 12-antenna with triple polarization characteristics operating in the frequency range of 3.4-3.6 GHz. Isolation between antennas is achieved by utilizing the orthogonal polarization technique. [6] A planar dual-band 4-antenna MIMO configuration for 5G mobile communication was proposed in which each planar folded monopole radiating element was surrounded by L-shaped meta-rim extended ground stubs. The antenna has multiple-resonances to get dual-band coverage from 1.6 to 3.6 and 4.1 to $6.1 \mathrm{GHz}$, Along with other characteristics, SAR also lies in the permissible range making it suitable for 5G mobile phone applications. [7] An eight-antenna multi-input multi-output (MIMO) array with high isolation, operating in the 3.5-GHz band was proposed. The novelty was that an array of balanced open slot antenna was designed, that enhanced the isolation between the adjacent port of the antenna. Furthermore, the coupling between the antennas was reduced by developing polarization diversity. Effects of radiation on hand phantom were also considered.[8] An eight-port antenna array was proposed in which each antenna is placed symmetrically at the corners of the mainboard. Port isolation is improved and the correlation between the antennas is reduced by using an orthogonal polarized square loop radiating strip. [9] The authors have given suggestions for designing the antennas for 5G cellular phones as 
higher screen-to-body ratios, larger battery sizes, antenna efficiencies, isolation levels, ECCs, ways of holding phones in landscape or portrait mode, compatibility with $2 \mathrm{G}$ to $4 \mathrm{G}$ technologies.[10][11] To reduce the size of the antenna, vertical stubs were introduced into the original self-isolated antenna. [12] An antenna covering as many as 13 bands from $2 \mathrm{G}$ to $5 \mathrm{G}$ and WLAN technologies in the metal-framed environment was proposed and the effect of the human hand was also studied on the antenna performance.[13] In a document released by Ericsson, the need and the advantages for combining 5G NR with LTE was explained as it would be a future-proof and costefficient way of improving performance and delivering the new 5G services to the subscribers. [14] A four-element MIMO configuration was proposed with SRR-loaded Slot-Loops for getting multi-band performance in the range 3 to $4 \mathrm{GHz}$ of $5 \mathrm{G}$ communication system.[15]

In the above discussions, 2 to 8 port MIMO systems are very much common and a lot of systems have been designed with the above-mentioned number of antennas. In the article, a 2-element, dual-band, dual-polarized slot antenna system is proposed that will support a $3.6 \mathrm{GHz}$ band of $5 \mathrm{G}$ mobile communication and $5.5 \mathrm{GHz}$ band for WLAN services that is extension of my previous work.[17] Both frequencies are independent of each other, which is considered an important feature while designing multi-band antennas. Good isolation is achieved by the physical separation between antennas and by using a parasitic patch. The antenna has good gain and efficiency so as to be considered as a candidate for antennas for future $5 \mathrm{G}$ smartphones.

\section{Antenna Design}

The transparent structure of the antenna is shown in fig. 1. It is $30 \mathrm{~mm} \times 30 \mathrm{~mm}$ antenna with two feed lines and two slots. The resonant frequency of the antenna depends on the perimeter of the squared slot. The dielectric wavelength for the first resonant frequency corresponds to $(\mathrm{a}+\mathrm{s}) / 2$ and for the second resonant frequency corresponds to $(\mathrm{c}+\mathrm{s}) / 2$. [16] The width of slot (s) is $0.375 \mathrm{~mm}$. The square slots in the ground structure are designed and optimized to get the desired resonant frequencies at $3.6 \mathrm{GHz}$ and $5.5 \mathrm{GHz}$. The optimized design parameters for the basic antenna design are given in table I. The design has a linear parasitic patch on the front side of the antenna placed diagonally between the two feed lines as shown in the transparent view of the antenna.

Each antenna is a linearly polarized antenna and placed in a perpendicular direction with respect to each other. The placement of the antenna feed resulted in the dual-polarization of the antenna system.

TABLE I. DESIGN PARAMETERS VALUES

\begin{tabular}{|l|l|}
\hline Parameter & Value (mm) \\
\hline $\mathrm{a}$ & 14 \\
\hline $\mathrm{b}$ & 13.75 \\
\hline $\mathrm{c}$ & 9.6 \\
\hline $\mathrm{d}$ & 8.85 \\
\hline $\mathrm{h}$ & 1.6 \\
\hline $\mathrm{Lf}$ & 11.4 \\
\hline $\mathrm{Li}$ & 11 \\
\hline Ls & 30 \\
\hline $\mathrm{s}$ & 0.375 \\
\hline Wf & 3 \\
\hline Wi & 0.5 \\
\hline Ws & 30 \\
\hline
\end{tabular}

\section{Results and Discussion}

The S-parameter results for the antenna are shown in fig. 4. The antenna resonates at $3.6 \mathrm{GHz}$ and $5.5 \mathrm{GHz}$ for ports 1 and 2 and because of the identical placement, the S-parameters are also identical. The $6 \mathrm{~dB}$ bandwidth for the antenna in the lower band is $450 \mathrm{MHz}(3.35$ to $3.8 \mathrm{GHz})$ and $600 \mathrm{MHz}(5.3$ to $5.9 \mathrm{GHz})$ in the higher band of operation. The isolation for the $3.6 \mathrm{GHz}$ band is more than $15 \mathrm{~dB}$ and for the $5.5 \mathrm{GHz}$ band, it is more than $11 \mathrm{~dB}$. The perpendicular orientation of feed lines is also responsible for isolation greater than $11 \mathrm{~dB}$. The threshold value considered for isolation is $10 \mathrm{~dB}$ as in the case of mobile antennas, the return loss of more than $6 \mathrm{~dB}$ is acceptable and the bandwidth is also calculated for the same level. 


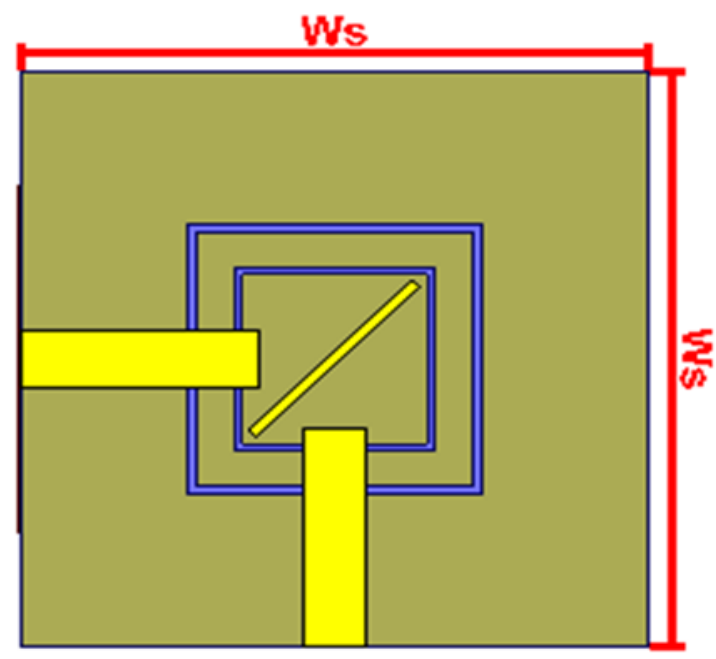

Fig. 1. Transparent view of proposed slot antenna

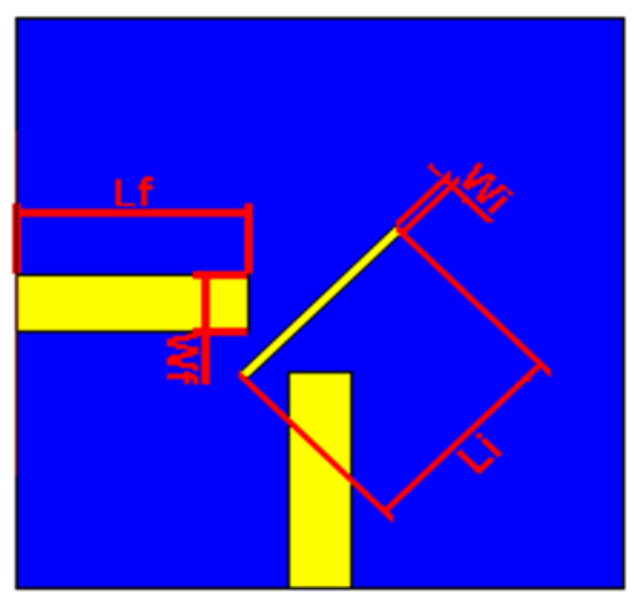

Fig. 2. Front view of the proposed slot antenna

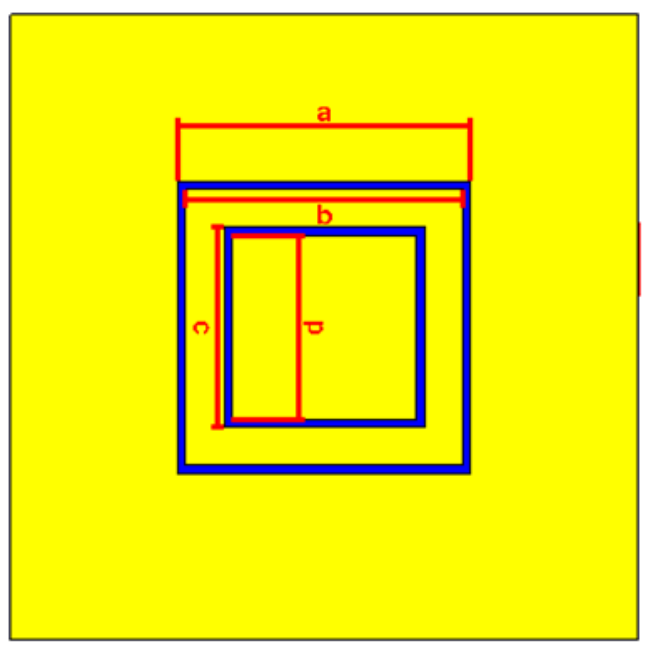

Fig. 3. Back view of the proposed slot antenna 


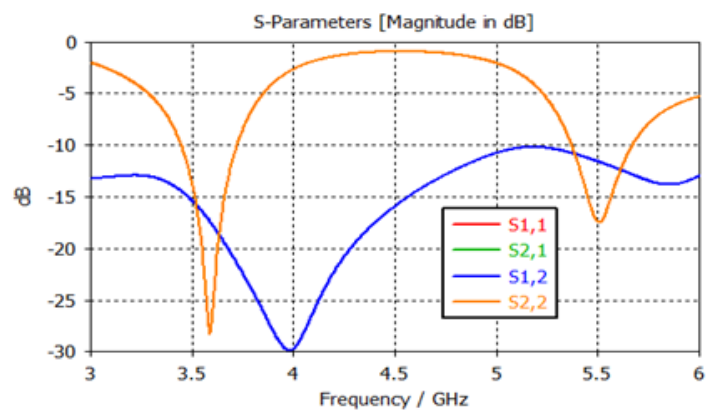

Fig. 4. S-parameter results for proposed antenna for ports 1 and 2

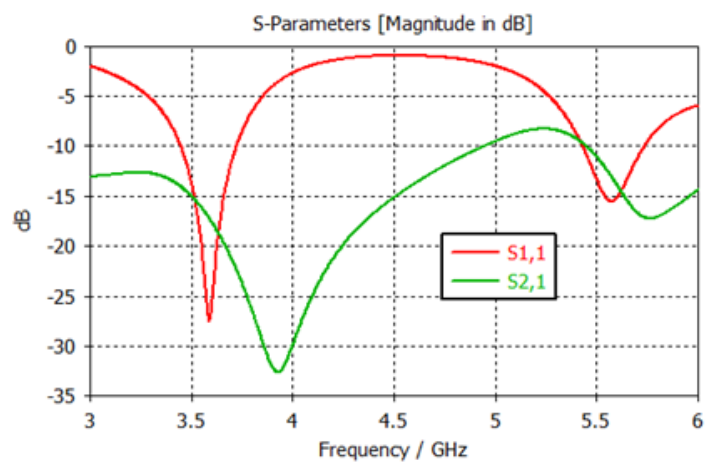

Fig.5. S-parameter results for proposed antenna for ports 1 and 2 without parasitic patch

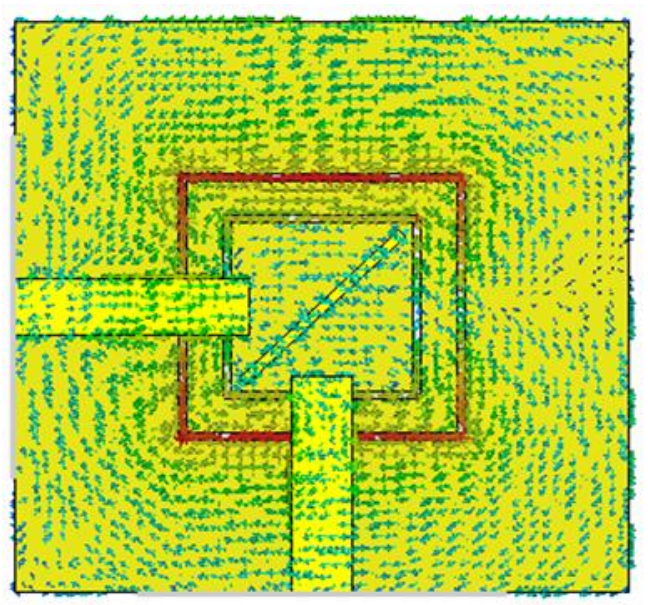

Fig.6. Current Distribution at $3.6 \mathrm{GHz}$ for port 1

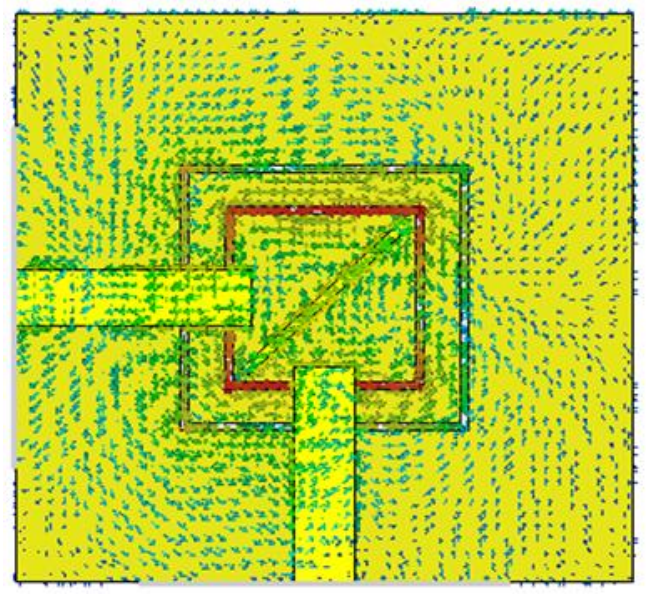

Fig.7. Current Distribution at $5.5 \mathrm{GHz}$ for port 1 


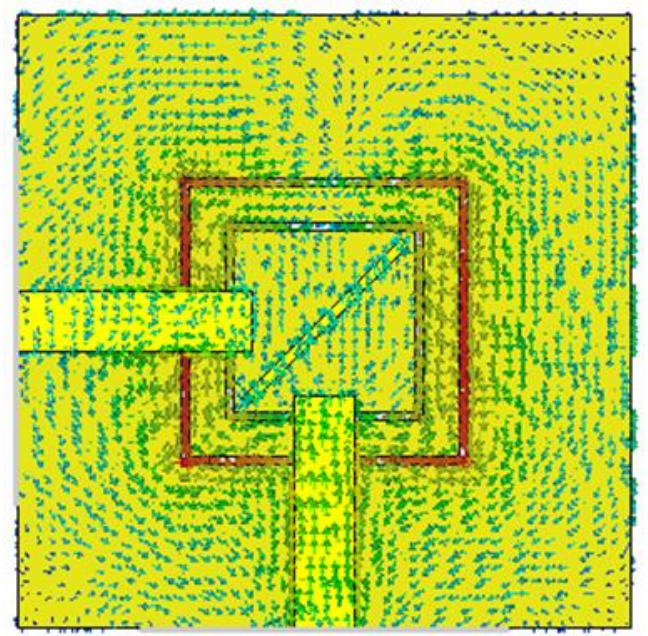

Fig.8. Current Distribution at $3.6 \mathrm{GHz}$ for port 2

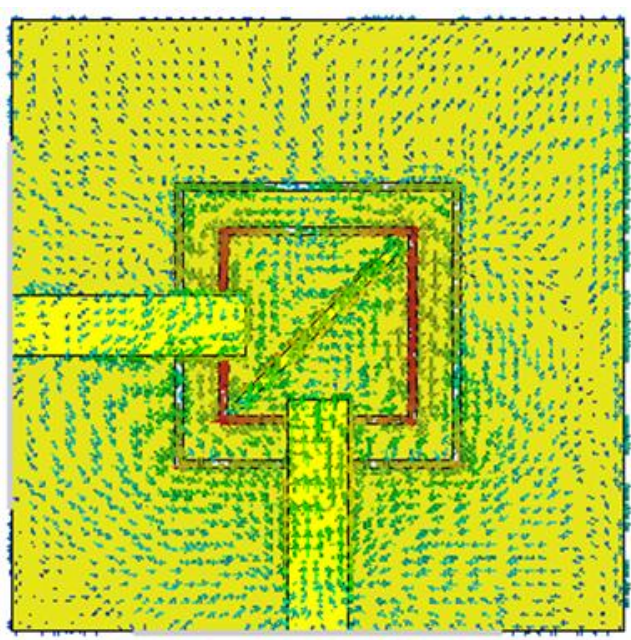

Fig.9. Current Distribution at $5.5 \mathrm{GHz}$ for port 2

Since both antenna elements are so closely placed that it resulted in the mutual coupling between the antennas particularly in the $5.5 \mathrm{GHz}$ as shown in the figure. To reduce the mutual coupling, a rectangular patch is diagonally placed between the two feed-lines. The s-parameter results without parasitic patch are shown in fig. 5

The current distribution for the antenna is shown in fig. 6 and fig. 7. for port 1 and in fig 8. and fig. 9. for port 2. It is clearly visible in fig. 6 and fig. 8 that at $3.6 \mathrm{GHz}$ frequency, the outer slot is radiating as the current density is higher and can be seen as red color over the outer slot and the same is observed for the inner slot at $5.5 \mathrm{GHz}$ frequency in fig. 7 and fig.8.

The results of variations in the antenna design parameters were also simulated. The s-parameters result for variation in the outer slot length ' $a$ ' is shown in fig. 10. and for inner slot length, 'c' is shown in figure 11 . It is verified that resonant frequency is inversely proportional the slot length, i.e. the resonant frequency decreases with an increase in slot length, with the perimeter of the slot. Both resonant frequencies are varying independently with the change in slot perimeter. The 3D radiation pattern for port 1 at $3.6 \mathrm{GHz}$ frequency is shown in fig.12 and at 5.5 $\mathrm{GHz}$ in fig.13. The shape of the radiation pattern is like a dumbbell, with a maximum gain of $2.06 \mathrm{dBi}$. The gain at $5.5 \mathrm{GHz}$, as seen from fig. 13 is $2.71 \mathrm{dBi}$. The radiation pattern and values of gain at both frequencies are the same for both ports of the antenna. The polar plot of the gain at $3.36 \mathrm{GHz}$ and $5.5 \mathrm{GHz}$ are shown in fig.14 and fig.15 respectively. 


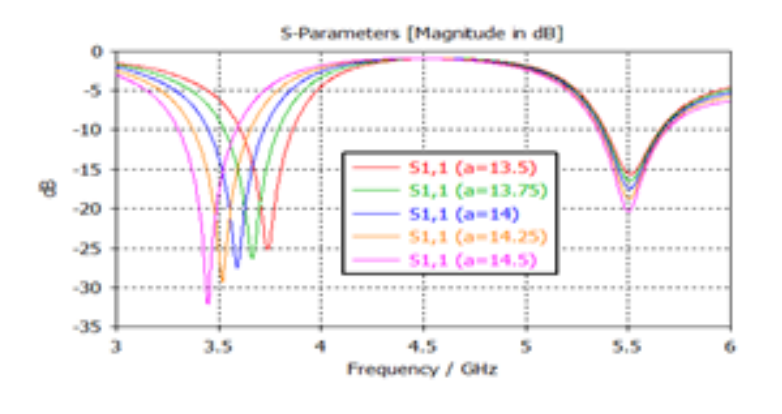

Fig.10. Effect of variation of outer slot length ' $a$ '

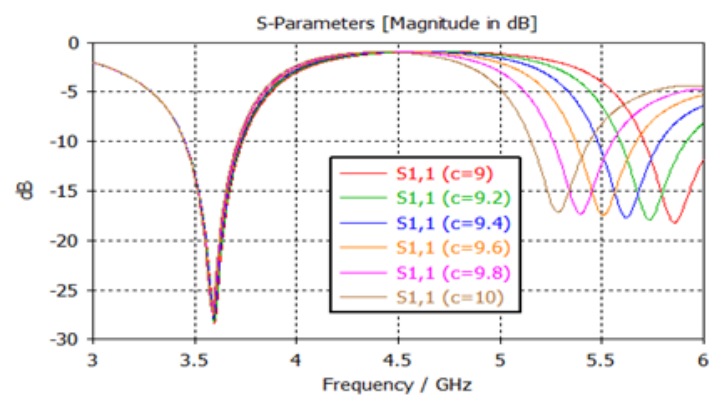

Fig.11. Effect of variation of inner slot length 'c'

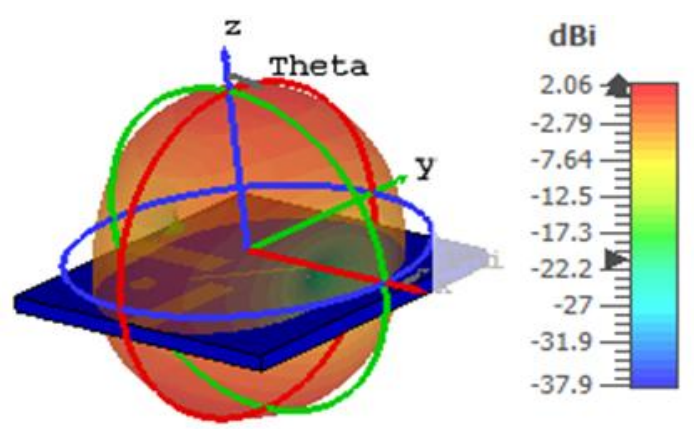

Fig.12. 3D radiation pattern for port 1 at $3.6 \mathrm{GHz}$

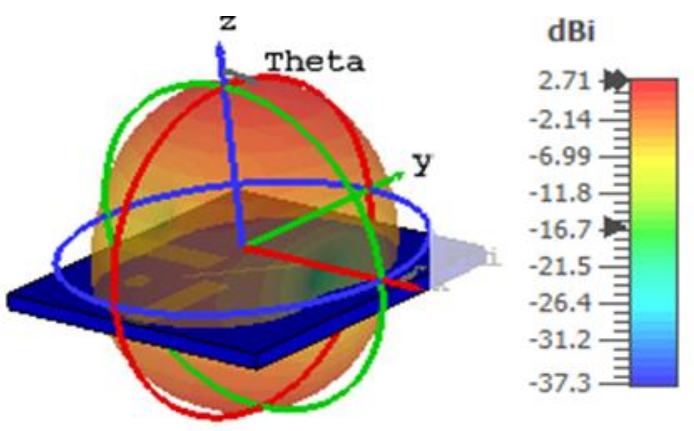

Fig.13. 3D radiation pattern for port 1 at $5.5 \mathrm{GHz}$ 
Farfield Gain Abs (Phi=90)

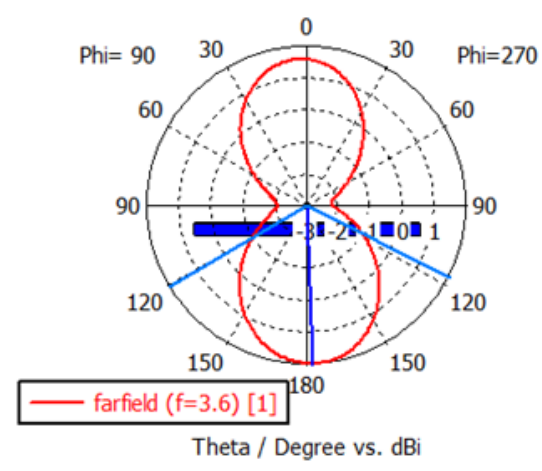

Fig.14. Polar plot of antenna radiation pattern at $3.6 \mathrm{GHz}$

Farfield Gain Abs (Phi=90)

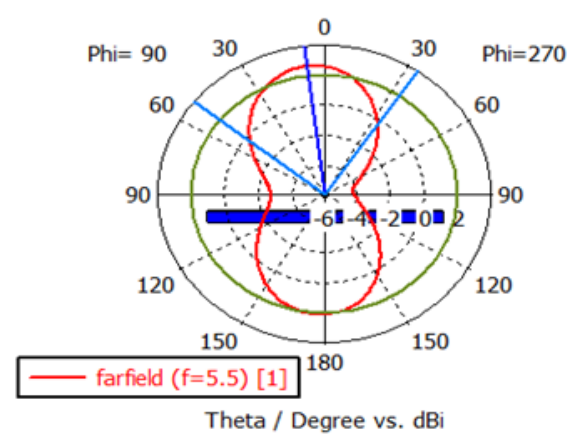

Fig.15. Polar plot of antenna radiation pattern at $5.5 \mathrm{GHz}$

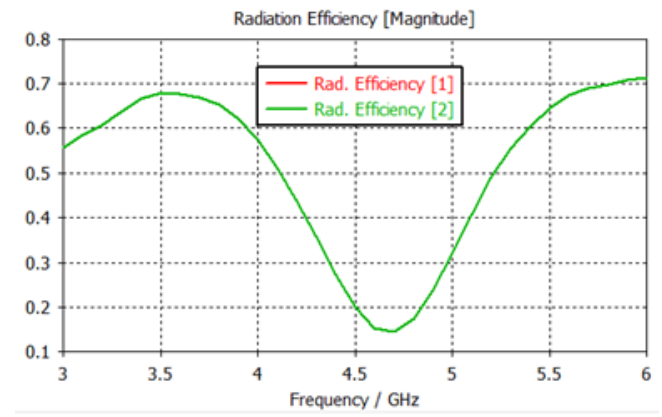

Fig.16. Radiation efficiency of the antenna for Ports 1 and 2

Fig.16. shows the radiation efficiency for the antenna for port 1 and port 2 . The value of the radiation efficiency is more than $65 \%$ in both operating for both ports.

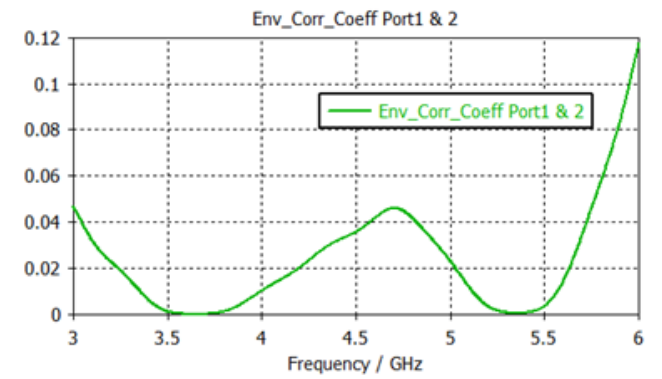

Fig.17. Envelope correlation coefficient for Ports 1 and 2 


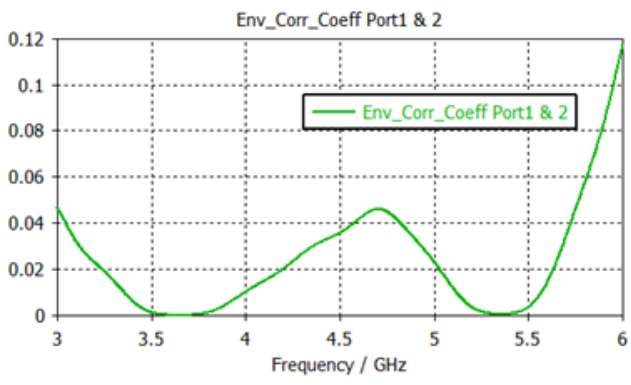

Fig.18. Diversity gain for Ports 1 and 2

Since this antenna is proposed as a MIMO antenna, so the parameters like Envelope correlation coefficient (ECC), diversity gain from s-parameters using post-processing are also calculated. Fig. 17 shows the variation of the Envelope correlation coefficient with respect to frequency and fig.18 depicts diversity gain for port 1 and port 2 of the antenna system. The value of ECC never goes beyond 0.1 and the value of diversity gain is almost 10 in the 3.6 $\mathrm{GHz}$ band and more the 9.5 in the upper-frequency band.

\section{Conclusion}

In this paper, a two-element dual-band MIMO antenna with dual-polarization characteristics for 5G user equipment are presented. Both operating frequencies $3.6 \mathrm{GHz}$ and $5.5 \mathrm{GHz}$ are independent of each other and the antenna has good gain, efficiency, sufficient isolation. The polarization of the antenna depends on the position of the feed elements on the substrate. The antenna is designed on FR-4 the substrate to ensure its easy availability and keeping it cost-effective. The resultant radiation pattern of the antenna set is bi-directional with low ECC and high diversity gain.

In future work, the number of elements will be increased as the design will done on the larger substrate of the size of PCB for smartphones

\section{Acknowledgement}

I would like to sincerely thank Mr. Sayyed Arif Ali and A.Z. Rizvi for helping me in documenting my work and providing me the guidance for future aspects of this initial work. I would also like to thank Mr. Mohd. Gulzar Ali, student, Mewar University for helping me in fabrication of the antenna.

\section{References}

Y. Huo, X. Dong, and W. Xu, "5G cellular user equipment: From theory to practical hardware design," IEEE Access, vol. 5, no. c, pp. 13992-14010, 2017.

A. Alieldin et al., "A Triple-Band Dual-Polarized Indoor Base Station Antenna for 2G, 3G, 4G and Sub-6 GHz 5G Applications,” IEEE Access, vol. 6, no. c, pp. 49209-49216, 2018.

A. Zhao, Z. Ren, and S. Wu, "Broadband MIMO Antenna System for 5G Operations in Mobile Phones," Int. J. RF Microw. Comput. Eng., vol. 29, no. 10, pp. 1-10, 2019.

L. Y. Rao and C. J. Tsai, "8-Loop Antenna Array in the 5 Inches Size Smartphone for 5G Communication the 3.4 GHz-3.6 GHz Band MIMO Operation,” in Progress in Electromagnetics Research Symposium, 2018 , vol. 2018-Augus, pp. 1995-1999.

Y. Li, C.-Y.-D. Sim, Y. Luo, and G. Yang, "Metal-frame-integrated eight-element multiple-input multiple-output antenna array in the long term evolution bands 41/42/43 for fifth generation smartphones," Int. J. RF Microw. Comput. Eng., vol. 29, no. 1, p. e21495, Jan. 2019.

M. Y. Li, Y. L. Ban, Z. Q. Xu, J. Guo, and Z. F. Yu, "Tri-Polarized 12-Antenna MIMO Array for Future 5G Smartphone Applications," IEEE Access, vol. 6, pp. 6160-6170, 2018.

J. Duan, K. Xu, X. Li, S. Chen, P. Zhao, and G. Wang, "Dual-band and enhanced-isolation MIMO antenna with L-shaped meta-rim extended ground stubs for $5 \mathrm{G}$ mobile handsets," Int. J. RF Microw. Comput. Eng., vol. 29, no. 8, pp. 1-8, Aug. 2019.

Y. Li, C.-Y.-D. Sim, Y. Luo, and G. Yang, "High-Isolation 3.5 GHz Eight-Antenna MIMO Array Using Balanced Open-Slot Antenna Element for 5G Smartphones," IEEE Trans. Antennas Propag., vol. 67, no. 6, pp. 3820-3830, Jun. 2019.

M. Li, Z. Xu, Y. Ban, C. Sim, and Z. Yu, "Eight-port orthogonally dual-polarised MIMO antennas using loop structures for 5G smartphone," IET Microwaves, Antennas Propag., vol. 11, no. 12, pp. 1810-1816, 2017.

H.-C. Huang, "Overview of antenna designs and considerations in 5G cellular phones," in 2018 International Workshop on Antenna Technology (iWAT), 2018, pp. 1-4.

W. Hong, "Solving the 5G Mobile Antenna Puzzle: Assessing Future Directions for the 5G Mobile Antenna Paradigm Shift,” IEEE Microw. Mag., vol. 18, no. 7, pp. 86-102, Nov. 2017. 
A. Zhao and Z. Ren, "Size Reduction of Self-Isolated MIMO Antenna System for 5G Mobile Phone Applications,” IEEE Antennas Wirel. Propag. Lett., vol. 18, no. 1, pp. 152-156, 2019.

D. Huang, Z. Du, and Y. Wang, "Compact thirteen-band antenna for 4G/5G/WLAN metal frame mobile phones,” Int. J. RF Microw. Comput. Eng., vol. 30, no. 3, pp. 1-10, 2020.

A. T. E. Sites, “Combining 5G NR with LTE," pp. 1-12.

D. Sarkar and K. V. Srivastava, "Four Element Dual-band Sub-6 GHz 5G MIMO Antenna Using SRR-loaded Slot-Loops," in 2018 5th IEEE Uttar Pradesh Section International Conference on Electrical, Electronics and Computer Engineering (UPCON), 2018, pp. 1-5.

N. O. Parchin, H. J. Basherlou, Y. I. A. Al-yasir, and A. Ullah, "Multi-Band MIMO Antenna Design with UserImpact Investigation for 4G and 5G Mobile Terminals," pp. 1-16, 2019.

A. Agarwal and J. Vaswani, "A FOUR PORT , DUAL BAND ANTENNA FOR FIFTH GENERATION MOBILE COMMUNICATION AND WLAN SERVICES,” ACTA Tech. CORVINIENSIS - Bull. Eng., vol. 4, no. XIII, pp. 73-76, 2020 